\title{
LA REITERANCIA DE LA CONDUCTA DELICTIVA EN ADOLESCENTES Y SU RELACIÓN CON LAS REPRESENTACIONES SOCIALES ACERCA DEL ROL EJERCIDO POR LAS MADRES
}

\section{THE REPEATED CRIMINAL BEHAVIOR IN ADOLESCENTS AND ITS RELATION TO THE SOCIAL REPRESENTATIONS ABOUT THE ROLE OF THEIR MOTHERS}

DOI: 10.22199/S07187475.2014.0001.00004

Recibido: 24 de Enero del 2014 | Aceptado: 28 de Marzo del 2014

MONICA VALGAÑON 1 ; LILIANA MUÑOZ 2 ; MARCELO BRICCOLA 3

(UNIVERSIDAD DE ACONCAGUA, Mendoza, Argentina)

\section{RESUMEN}

Introducción: La reiterancia es aquella conducta transgresora de la ley repetida en más de dos oportunidades en la que no se produjo condena por ninguna de las infracciones, en función de que quien las comete es menor de edad, es decir menor de 18 años. Las trasgresiones se registran en el micro legajo que obra en Comisaría del Menor donde se labran las actuaciones administrativas, 0 en los expedientes de los Juzgados Penales de Menores lo que no es considerado como "prontuario delictivo". Objetivo: Se busca relacionar la representación social del rol parental con la conducta delictiva como pauta y la posible historia de vulneración de derechos de los jóvenes reiterantes. Método: Se trabajó con la casuística registrada en la Comisaría del Menor, cotejada con los ingresos en el Sistema Penal Juvenil, durante los últimos seis meses del año 2012. Los instrumentos utilizados fueron el Test de Evocaciones Jerarquizadas (Abric 2001, Mazitelli 2007 y Gonzalez, 2010), la Escala de Competencias Parentales Percibidas (adaptada) (Bayot, Hernandez Viadel y Julian L.F., 2005), más una entrevista focalizada a expertos y el estudio de legajos institucionales. Resultados: el rol parental de los adolescentes reiterantes se basó en una representación social idealizada, que destaca afectos positivos que no son palpables en el ejercicio concreto de la parentalidad. A su vez los jóvenes padecieron en su historia vital grave, continua y simultánea vulneración de derechos básicos. Conclusión: Las madres de los adolescentes infractores estudiadas, adhieren a una representación social afectiva, positiva de la parentalidad, expresan disfunción en el ejercicio concreto, incompetencias en las tareas normativas y suplen las carencias con sustitutos institucionales de manera parcial. La disfunción parental se agrega a la vulneración de derechos continua en la vida de los adolescentes.

PALABRAS CLAVE: Reiterancia, conducta delictiva, representación social, parentalidad, familia.

\section{ABSTRACT}

Introduction: reiterancia is that transgressive behavior repeated law in more than two occasions in which there was no conviction for any offenses, according to the party who commits a minor, ie under 18 . The transgressions are recorded in the micro file that works in Precinct Menor where administrative proceedings are worked, or in the records of the Criminal Courts Juvenile what is not considered " criminal record ". Objective: The aim is to relate the social representation of the parental role in the criminal conduct as a guideline and possible history of violations of rights of young people returning. Method: . Worked with casuistry registered at the police station Minor, collated with revenues in the Juvenile System, for the last six months of 2012 The instruments used were the Test of Nested Evocations ( Abric 2001 Mazitelli 2007 and Gonzalez , 2010), the Scale of Perceived Parental Competencies (adapted) ( Bayot Hernandez Viadel and Julian LF, 2005 ), plus a focused interview experts and studying institutional bundles. Results: The parental role of returning adolescents was based on an idealized social representation that highlights positive affects that are not evident in the practical exercise of parenthood. In turn young people suffered in his grave, continuous and simultaneous violation of basic rights vital history. Conclusion : Mothers of young offenders studied, adhere to a loving, positive social representation Parents express dysfunction in the concrete exercise , incompetence in policy work and compensate the lack institutional substitutes partially. The parental dysfunction is added to the violation continuing rights in the lives of adolescents.

KEY WORDS: Reinterancy, criminal behavior, social representation, parenting, family.

\footnotetext{
2. Insitituto de Investigaciones, Universidad de Aconcagua. E-mail: lilianamgaldame@hotmail.com

3. Insitituto de Investigaciones, Universidad de Aconcagua. E-mail: marcebriccola@yahoo.com.ar
}

(1) Aconcagua. E-mail:monicabeatrizvalganon@gmail.com 


\section{INTRODUCCIÓN}

En las sociedades modernas las funciones de la familia se han reducido y transferido no por el ocaso familiar, sino por una creciente especialización y diferenciación de los núcleos de convivencia (Donini, 2007). En este contexto surge el ejercicio de la paternidad co-ejercida con otros 0 con instituciones prestadoras de servicio, tales como guarderías, centros maternales, abuelos, niñeras, instituciones educativas, etc.

La investigación criminológica incide en el importante rol de las instituciones de socialización en la evitación de la conducta delictiva, resaltando el papel de la familia como crucial para explicar el comportamiento adaptado. Así, a pesar de todos los cambios, la familia sigue siendo el agente más importante de socialización, aportando con un entorno de intimidad, donde ideas, afectos y sentimientos se aprenden e intercambian, al mismo tiempo que conforma un reflejo de la sociedad (Musitu \& Buelga 2001; Rodríguez y Torrente, 2003).

La socialización aparece como el proceso a través del cual el ser humano adquiere un sentido de identidad personal y aprende las creencias y normas de comportamiento, valoradas y esperadas por las personas que le rodean (Chan Gamboa, 2006). Es entendida como un conjunto de relaciones, una forma de vivir juntos y de satisfacer necesidades emocionales mediante la interacción de sus miembros, que junto con el amor, el odio, la diversión y la violencia, constituye un entorno emocional en el que cada individuo aprende las habilidades que determinarán su interacción con otros en el mundo al que se adapta (Chan Gamboa, 2006). Este proceso no finaliza en la niñez; continúa durante la adolescencia, donde los cambios evolutivos -biológicos, cognitivos y emocionales-, asi como los contextuales que sufre el adolescente, requieren que se produzca una reformulación, existiendo consenso acerca de la asociación entre estilo parental y consecuencias en el desarrollo psicosocial del hijo (Musitu, Buelga, Lila y Cava, 2001).

Para García Linares, Pelegrina \& Lendínez (2002) los hijos, instrumentalmente competentes, son producto de hogares en los que los padres se comportan de una determinada manera (padres afectivos, establecimiento de normas racionales y claras a la vez que permiten al hijo autonomía dentro de límites, capacidad para comunicar con claridad sus expectativas) . Frente a ello, las historias de vida de los menores infractores presentan un alto índice de hogares en los que frecuentemente se observa la ausencia de las figuras parentales, presencia de padres $y$ hermanos con antecedentes penales, falta de armonía familiar, privación socioeconómica, el estilo educativo negligente y un control poco consistente, desempleo, entre otros (Gonzalez y Gonzalez, 2008; Demuth y Peñalva 2001; Farrington, 2000, 2002; Paíno y Rodríguez, 1998; Rutter,Giller y Hagell, 1998;).

La investigación criminológica actual ha puesto de manifiesto que los adolescentes, en comparación con niños y adultos, se implican con más probabilidad en comportamientos temerarios, ilegales $y$ antisociales (Musitu, 2002). Igualmente, es más probable que causen alteraciones del orden social y que se impliquen en conductas potencialmente perjudiciales para ellos mismos o para las personas que les rodean. Así, la adolescencia y primera juventud son los periodos donde más elevada es la prevalencia de una variedad de conductas de riesgo e incrementan entre cinco y seis veces -como, por ejemplo, conductas delictivas, conductas violentas, consumo de sustancias, conducción temeraria y conducta sexual de riesgo (Musitu, 2002; Rodríguez y Torrente, 2003; Grossi, Paino, Fernandez y Rodriguez 2000, Becedoniz, Rodriguez, menendez, Bringas, 
Balaña y Paino 2006). De ahí la relevancia de considerar la vinculación de la reiterancia de la conducta ya no transgresora simplemente como patrón esperable en el período de la adolescencia, sino como conducta delictiva, que causa un daño discernible y objetivo en la vida y bienes de otras personas del entorno. Dicha conducta delictiva se estaría estableciendo como una modalidad relacional del joven, y estaría favorecida por su núcleo familiar primario.

El pensamiento social sobre el ejercicio de la parentalidad y la competencia parental serían factores ineludiblemente relacionados con la conducta delictiva.

\section{Acerca de las Representaciones Sociales}

Conceptualmente, la representación social se refiere a la:

Actividad mental que despliegan los individuos y grupos con el fin de fijar su posición en relación con situaciones, acontecimientos, objetos $y$ comunicaciones que les conciernen de la vida cotidiana y que se encuentran en la base de la construcción de una realidad social de orden consensual. (Jodelet, 1993 p.473)

Para Moscovici y Hewstone (1986), son teorías basadas en el sentido común y en esta función muestran cómo suceden las cosas. Dicho de otra forma tienen como misión describir, clasificar y explicar; sin embargo, a diferencia del conocimiento generado a partir de la ciencia, las representaciones sociales rápidamente trasuntan explicaciones acerca de los eventos de la vida cotidiana, explicaciones fuertemente arraigadas al minuto de contactarse con la realidad social.

Las representaciones tienden un puente entre el individuo, la cultura y la historia, es decir entre la subjetividad y la vida social de los individuos. Se relacionan, consecuentemente con los contenidos del pensamiento cotidiano, refiriéndose específicamente a las imágenes y modelos explicativos que un determinado grupo social tiene acerca del algún fenómeno de la realidad.

En este marco, consideraremos las representaciones sociales como fenómenos sociocognitivos, organizaciones significantes y estructuradas, desde contextos y circunstancias concretas e inmediatas. Estas representaciones interaccionan con factores generales como lo ideológico, el contexto social, lugar que ocupa el individuo o el grupo en la sociedad; y aparecen como orientadoras de acciones, conductas, relaciones sociales y determinantes de anticipaciones y expectativas, como principios generadores de toma de posición, ligados a inserciones sociales. Su significación está determinada y formulada por un contexto discursivo (son las producciones discursivas las que permiten conocer la representación) y el contexto social (anclada en significaciones más amplias y generales del campo social).

Parentalidad, familias y delitos en la adolescencia

Musitu y García, (2004) han constatado que un ambiente familiar positivo, caracterizado por una comunicación abierta y por la presencia de afecto y apoyo entre padres e hijos, es uno de lo más importantes garantes de bienestar psicosocial en la adolescencia, mientras que un ambiente familiar negativo con frecuentes conflictos y tensiones, se dificulta el buen desarrollo de los hijos y aumenta la probabilidad de que surjan problemas de disciplina y conducta (Dekovic, Wissink y Mejier, 2004).

Esto es muchas veces observable cuando se trabaja con familias que tienen problemas con los hijos adolescentes, que realizan infracciones a la ley. Es decir, hay un gran porcentaje de ellas, que tienen características negativas, como se menciona anteriormente. 
La familia tiene todavía el rol primordial de transmitir a sus hijos una serie de creencias, valores y normas que les ayudarán a convivir en la sociedad de la que forman parte, lo que se conoce como socialización.

A través de la socialización, las personas aprendemos los códigos de conducta de nuestra sociedad, nos adaptamos a ellos y los respetamos para el buen funcionamiento social (Paterna, Martínez y Vera, 2003). La familia es un contexto especialmente privilegiado para el aprendizaje de estos elementos sociales y culturales, aprendizaje que tiene lugar principalmente durante la infancia y la adolescencia.

Pero no todos los individuos tenemos la destreza para el óptimo desarrollo de la parentalidad. López, Cabrera, Quintana y Maíquez (2009), estudiaron las competencias parentales en contextos de riesgo. Ellos proponen una serie de variables como las habilidades educativas, de gestión de autonomía y apoyo social, para la vida personal, para la organización doméstica como base de las competencias. Proponen una serie de recomendaciones para la evaluación de las competencias.

La parentalidad puede diferenciarse en biológica y social. La primera es el resultado biológico de la procreación y la segunda alude al cuidado, educación y protección de los hijos. Las habilidades prácticas que permiten el desarrollo sano de los hijos, son consideradas como "competencias parentales". Las necesidades que deben satisfacer son múltiples y cambian con el desarrollo evolutivo y el entorno social (Badury \& Dartagnan, 2005, 2010; Valgañón 2012, Muñoz, 2012).

La capacidad de apego a los hijos, la empatía, los modelos de crianza y la capacidad de utilizar las redes sociales y comunitarias son considerados aspectos centrales, evaluables de las competencias parentales y pueden ser ejercidas aun por quienes no tienen parentesco con los hijos (Musitu, Estevez, Jimenez, Herrero 2007). Los estilos de crianza vivenciados durante la niñez suelen ser buenos predictores del ajuste en la adolescencia (Casullo y Fernandez Liporace, 2008).

Las familias con dificultades en la socialización y en el control social, suelen estar a cargo de progenitores con fallas en las habilidades y competencias familiares. El nivel educativo y cultural de los progenitores, sería un aspecto relacionado con el proceso de maduración, ya que un nivel bajo de los padres condicionará el aprendizaje de los hijos. No es posible transmitir aquello que no se sabe, que no se practica; en una palabra, aquello en lo que no se es competente (Garrido y Martínez, 1998; Fernández Ríos, Gómez Fraguela y Rodríguez, 1999).

El contexto social, económico y cultural en el que está ubicada la familia puede facilitar la situación de marginación 0 exclusión de cierto contexto normativo y la adaptación a otro medio, en el que podría resultar sincrónica la conducta delictiva y cierta modalidad de ejercicio parental. Planteamos, así, que la familia es el primer agente socializador encargado de transmitir las normas, costumbres y valores sociales (Henggeler, 1989), ejerciendo una influencia sobre las conductas de sus descendientes (Ryan \& Reading 2004), y dependiendo de la distancia, entre el grupo al que pertenece la familia y el grupo normativo, esos valores coincidirán o no (López Coira, 1987; Rodríguez y Grossi, 1999; Vazquez Gonzalez 2003, Vega, 1987). No podemos, pues, considerar los conceptos de conducta desviada y conducta delictiva como sinónimos. En este sentido, la conducta delictiva la definimos como aquella conducta antisocial que interfiere con los derechos de otras personas o amenaza el bienestar del propio individuo 0 de la comunidad (Departament of Health, Education and Welfare, 1972, citado por Dussich, 1989); y 
denota violación de las normas formales, de la ley (Dussich, 1989; Kauffman,1993) del grupo normativo al que se pertenece.

Por lo expuesto precedentemente este artículo propone conocer las representaciones sociales acerca del rol parental, desarrollado por las madres de los adolescentes con reiterancia delictiva y su relación con las competencias parentales de dichas madres.

\section{MÉTODO}

Se trata de un estudio exploratorio de tipo cualitativo, que utiliza como encuadre teórico y metodológico la teoría de las Representaciones Sociales, aplicadas al ejercicio del rol parental en madres de adolescentes reiterantes en la conducta delictiva.

El supuesto considera que las representaciones sociales acerca del rol ejercido por las madres coinciden con las competencias parentales desarrolladas hasta el momento, las cuales serían ineficaces para el control de la conducta social adecuada de los menores reiterantes.

\section{Participantes}

Fueron incorporadas al estudio madres de jóvenes menores de 18 años de edad, que durante los primeros seis meses del año registren características de reiterancia delictiva, en la Comisaría del Menor y estén sostenidos en el Sistema Penal Juvenil. Se trabajó con 20 madres e hijos ingresados en ese periodo, lo que corresponde a un $43 \%$ del total de ingresos por dicha causa.

\section{Procedimientos}

La recolección de datos se realizó caso por caso, realizando el circuito institucional pertinente todas las veces. Esto es, la toma de conocimiento semanal de los ingresos en Comisaría, el filtro de situaciones delictivas no pertinentes. Sólo se consideraron las causas "reiterantes" de robo 0 robo agravado.

Luego se individualizó la familia del joven internado y se solicitó su colaboración y consentimiento informado, para la toma de los instrumentos. Asimismo se procesaron los datos relativos a la vulneración de derechos, según la documentación hallada en los legajos institucionales.

Se observó que al momento de la toma del test de evocaciones, las personas tuvieron dificultades en producir la cantidad de palabras esperadas. Les costó emitir palabras o frases. Este dato será tenido en cuenta en el momento de interpretación de los datos.

En cuanto a la entrevista focalizada a expertos, consistió en la realización de cinco preguntas relativas a los patrones de crianza que los profesionales en su abordaje técnico han ido considerando presentes.

\section{Instrumentos.}

Test de evocaciones jerarquizadas (Graca et al. 2004; Mazzitelli, 2007, Gonzalez 2010.)

Esta es una técnica asociativa, cimentada en una producción de palabras 0 expresiones espontáneas, la que, como plantea Abric (2001) "...consiste, a partir de un término inductor (o una serie de términos), en pedir al sujeto que produzca todos los términos, expresiones o adjetivos que se le presente al espíritu." (p.59) que permitirá detectar, el contenido y la estructura de la RS de un determinado grupo (Abric, 2001; Mazzitelli, 2007; Petracci y Kornblit, 2007, Gonzalez 2010).

Abric (2001) expresa que la asociación libre tiene la característica de permitir el acceso a una producción espontánea, con una dimensión proyectiva, que al ser menos 
controlada y dirigida permitirá acceder, más rápido y fácilmente a los elementos que forman el universo semántico del término $u$ objeto estudiado. Además remarca que a partir de esta metodología se puede hacer explícito elementos latentes que suelen ser enmascarados en producciones discursivas. Mostrando así una de las ventajas de la técnica de asociación frente a los métodos interrogativos o de producción de discursos.

En función de lo expuesto para el presente estudio, hicimos uso de esta metodología asociativa a partir de la evocación jerarquizada, donde el mismo sujeto produjo los términos desde la evocación que la palabra "madre" le provocó, expresiones que él mismo jerarquizó desde su propia gestión y que permitió detectar el contenido de las RS y reconocer las asociaciones nucleares $u$ organizadoras de las que no lo son para cada sujeto.

Escala de competencias parentales percibidas (Bayot, Hernández, y De Julián 2005)

Se trata de la adaptación de los ítems y categorías que refieren al desempeño parental, según la versión de los padres. En ésta se requiere una breve respuesta, cuya organización obedece a patrones cognitivos, y es analizada según su contenido. La toma de la misma se realizó en los talleres padres de chicos en conflicto con la ley.

La codificación de los datos se realizó de manera nominal (presencia o ausencia del ítems y se consideró la particularidad de alguna respuesta.

\section{Estudio de legajos institucionales}

La historia de vida de los jóvenes se escribe en una carpeta de uso institucional. Tiene carácter de documento público, de acceso restringido, en la que se certifica la evolución integral del caso. Está íntegramente foliada y se registra toda novedad producida en el caso. Se tomó la información disponible al momento de su ingreso y la consignada en los últimos tres meses de vida institucional a la fecha del relevamiento. Se agruparon los datos formando las siguientes categorías que responden al tipo de vulnerabilidad vivida.

Con la documentación relevada, se construyeron tablas que describen la frecuencia de aparición de cada categoría, en cada legajo personal. Se le asignó una puntuación porcentual, como una licencia metodológica, que permite un mejor análisis y facilita la comparación entre las técnicas administradas.

\section{Entrevista focalizada a expertos}

Consistió en la realización de cinco preguntas a los profesionales que se desempeñan en el área asistencial del Sistema de Responsabilidad Penal Juvenil, con una antigüedad no menor a 5 años de trabajo y que se encuentran abocados al trabajo con jóvenes "reiterantes" por delitos de robo o robo agravado. Consistió en la autoadministración de las preguntas y su codificación posterior en un cuadro de doble entrada. Luego se sintetizó en la redacción de un perfil lo encontrado. Los datos relevados se cotejaron con lo encontrado en el estudio de legajos institucionales, obteniendo un perfil del estilo de crianza recibida.

\section{RESULTADOS}

\section{Test de evocaciones Jerarquizadas}

El resultado descripto en la Tabla $\mathrm{N}^{0} 1$ alude a que en el núcleo del pensamiento social del grupo acerca de qué es ser madre, se encuentran aquellas emociones con vivencia positiva que surgen del hecho mismo de tener un hijo. Ser feliz, sentir amor, alegría, ternura fueron algunas de las palabras de la categoría. En el eje central se encuentran aquellas condiciones personales que son consideradas 
necesarias para llevar a cabo una tarea parental funcional, como la protección, la empatía, la paciencia, etc.

TABLA 1.

Estructura de las Representaciones Sociales de la parentalidad en madres con hijos en reiterancia delictiva.

\begin{tabular}{lll}
\hline & Frecuencia alta & Frecuencia baja \\
\hline \multirow{3}{*}{$\begin{array}{ll}\text { Valor } \\
\text { alto }\end{array}$} & Aúcleo: & Elementos de \\
& Atributos & contraste \\
& personales & Expectativa \\
& parental \\
& Primera periferia: & Segunda \\
Valor & Deberes & periferia \\
bajo & asistenciales & Afectos \\
& Tareas normativas & negativos \\
& Deberes afectivos & \\
\hline
\end{tabular}

En la primera periferia, los deberes asistenciales (llevar al médico, comprar ropa, criar, estar, etc.), los deberes afectivos (ayudar, comprender, dar afecto, etc.) y las tareas relacionadas con la puesta de límites y parámetros de socialización completan la representación social del grupo.

Los afectos negativos (miedo, llanto, lucha, preocupación) adheridos a la parentalidad se describen muy alejados del núcleo de la representación, forman la segunda periferia.

Un simbolismo de contraste se evidencia en la expectativa parental, es decir que algunos padres del grupo estudiado expresaron que el hecho de ser padres puede asociarse al cumplimiento de algún deseo 0 ansia personal. Es llamativo que consideren al hijo con la expectativa de la "libertad", lo cual podría relacionarse con las condiciones desfavorables de vida en la que se desarrollan tanto padres como hijos.

El pensamiento social del grupo se puede relacionar con las similitudes que presenta el grupo de padres sin disfuncionalidad en el ejercicio, lo cual resulta contrastante y contradictorio con los resultados de la crianza, en la que los jóvenes padecen sostenidas vulneraciones de derechos, malos tratos, riesgos sociales y los resultados de la falta de cuidados y límites se expresan en la reiterancia delictiva. Los patrones de socialización y de ajuste a grupos normativos mayoritarios no son impuestos en la crianza de estos jóvenes, a pesar que el pensamiento social de estas madres pudiera corresponder al orden social.

La representación social de la parentalidad contrasta con los resultados del ejercicio de la tarea parental.

\section{Escala de competencias parentales}

De lo encontrado, se puede señalar que: en asunción del rol parental el $100 \%$ está de acuerdo y estimula para que cada miembro diga lo que piense y siente, enfatizando que la manera de estimular es la comunicación, no pudiendo precisar otros estímulos. Todas coincidieron que la llegada de los hijos fue un cambio estructural y emocional en la familia. Dicen felicitar a los hijos siempre que realizan conductas correctas, y una sola responde que no hace falta que los felicite a sus hijos ya que ellos saben que es lo correcto lo que han hecho. Más del "95\%" manifiesta respaldar en sus hogares las reglas, normas y expectativas de conductas de sus hijos en la escuela; una sola mamá responde no apoyar las reglas emanadas por la institución escolar.

Todas dicen conocer a los amigos de sus hijos, a pesar de que no van a la casa del joven, sólo seis (6) reciben a los amigos en la casa, otras seis (6) ocasionalmente y cinco (5) responden directamente que no. Sólo una sola responde que su hijo no sale de noche, el resto responde que si, la frecuencia de las salidas está ligada a cómo se encuentre el adolescente en esos tiempos, si está "colocado" (hacen referencia a que este en periodos donde consumen diferentes drogas) puede salir todos los días de noche, la mayoría a veces 
saben con quién sale de noche, coincidiendo la dificultad que tienen para que el adolescente cumpla con los horarios establecidos, algunos no regresan a los hogares por algunos días, no sabiendo la progenitora en qué lugar ni con quién están.

En el componente de ocio compartido se observa que la mayoría comparten algunos programas de TV y luego lo comentan, de los programas que mencionan ninguno hace referencia a documentales, noticieros, 0 alguno que fomente las capacidades cognitivas del grupo familiar. La mitad indica que además de ir la escuela, sus hijos se relacionan con otros niños como por ejemplo ir al parque, a la plaza 0 simplemente a la casa de algún familiar a donde hay niños también, la otra mitad responde que sus hijos no acuden a otros lugares que no sea la escuela, es decir que no se estimula el potencial para desarrollar lo relacional en los hijos. Con respecto a las cosas cotidianas que comparten, sólo 2 responden que no salen de la casa, el resto (18) expresa que van al parque, a comer, al río, una o dos veces al mes $u$ a otros lugares de esparcimiento familiar.

En asesoramiento y la orientación respecto de la sexualidad han sido ellas quienes informaron a sus hijos, luego la escuela, los amigos y por último los hermanos mayores. Manifiestan la totalidad de las madres, que sus hijos conocen de anticoncepción, a pesar de que hay en estas familias embarazos adolescentes, que los mismos son informados de los diferentes métodos anticonceptivos siendo las madres, en su mayoría, las encargadas de hacerlo, luego la escuela, después los hermanos, ocupando el mismo lugar el padre y los amigos.

En el componente de la implicación escolar todas las encuestadas coinciden de que asisten a la escuela de sus hijos, 10 revisan los cuadernos de sus hijos todos los días, 2 sólo lo hacen una vez por semana, 4 cuando hay alguna nota de la maestra y una expresa que sus hijos están grande para que le este revisando los carpetas.

Más del "85\%" expresa conocer los deberes y tareas que se les da a los hijos en la escuela contra alrededor de un $5 \%$ que dice desconocer esas tareas e igual porcentaje responde a veces estar al tanto. Coincidiendo que 19 de las 20 entrevistadas dijeron tener una óptima relación con las maestras, pudiendo recibir indicaciones por parte del personal de la escuela en caso de que fuera necesario para una evolución favorable en el rendimiento escolar; habiendo una contracción cuando responden acerca de realizar un plan de trabajo con el maestro para mejorar el rendimiento ya que 15 respondieron si y 5 no. Casi la totalidad responde que sus hijos no poseen maestras particulares, sólo dos alegan que si ya que asisten a establecimientos educativos que tienen apoyo escolar a contra turno del horario habitual de la escuela.

La mayoría consulta con las maestras acerca de le evolución que están teniendo sus hijos en el colegio, sólo dos de las encuestadas responden no consultar.

Con respecto a la dedicación personal sólo tres (3) de las encuestadas expresan no tener tiempo para dedicarles a los hijos, aduciendo el trabajo fuera del hogar, el resto de la población (17), responde que si le dedica tiempo a los hijos, incluso algunas respondieron que están "todo el día con ellos". En lo que respecta a los hábitos de higiene la mayoría establece una rutina diaria y les parece importante hacerlo, pero relatan que es difícil sostenerlo con el tiempo. Acotan que tienen la libreta sanitaria en condiciones, archivan documentación relevante de la prole, nueve (9) de las diecisiete (20) encuestadas llevan a los hijos cuando se enferman y ocho (8) para los controles médicos. La mayoría (10) respondió no haberle realizado tratamiento especial a alguno de sus hijos y el resto (7) 
expresan haber acudido a tratamiento por el tema de adicciones.

Otro de los ítems a responder hace referencia a la colaboración doméstica que poseen los hijos dentro de la familia, la mayoría responde que si. Ayudan a realizar algunos quehaceres domésticos como hacerse la cama, limpiar el patio, los platos, etc.

\section{Estudio de legajos institucionales}

La Tabla N $N^{0} 2$ muestra que al momento de la internación de los chicos, éstos habían sufrido simultáneamente diversos tipos de vulneración de derechos estando presente en el total de los casos la violencia familiar y el abandono de alguno de los progenitores. Han padecido la falta de asistencia médica y la desnutrición. Han sido sometidos a presenciar la comisión de delitos 0 a participar de éstos. Otro tipo de maltrato se expresa en la falta de inscripción legal de su identidad y que no se los incorpore al sistema educativo formal Han debido ejercer mendicidad $y$ han debido trabajar. El maltrato físico también se ha reportado, al igual que abuso sexual.

TABLA 2.

Frecuencia y porcentaje de tipo de vulneración de derechos vivida por los jóvenes.

\begin{tabular}{lll}
\hline \multicolumn{1}{c}{ Categoría* } & Frecuencia & Porcentaje \\
\hline Desnutrición & 16 & 88 \\
Falta de asistencia médica & 17 & 94 \\
Falta documento de identidad & 12 & 66 \\
Sin ingreso al sistema escolar & 11 & 61 \\
formal & & \\
Abandono parental & 18 & 100 \\
Testigo de violencia familiar & 18 & 100 \\
Ejercicio de mendicidad & 11 & 61 \\
Trabajo infantil & 9 & 50 \\
Testigo de delitos & 14 & 77 \\
Partícipe de delitos de los & 5 & 27 \\
adultos & 15 & 83 \\
Maltrato físico & 18 & 100 \\
Ingresos familiares inestables & 6 & 33 \\
Abuso sexual & (1) & \\
\hline *Nota: las categorías no son excluyentes, pueden estar presentes \\
de manera conjunta o separada en cada caso. N: 18.
\end{tabular}

\section{Entrevista focalizada a expertos}

De acuerdo a los resultados de la entrevista, se puede observar que éstos expresan que los jóvenes reciben afecto, sobre todo cuando son pequeños. Todos coinciden en señalar déficit en la puesta de límites, guías eficaces, controles normativos y procesos favorables de adaptación social. Dos de los cinco entrevistados relata que las tareas parentales que realizan funcionalmente están ligadas a las nutricias, como es el alimentarlos y dar afecto. No hay puesta de límites claros, concisos y sostenidos en el tiempo. Son apoyados por terceros de manera permanente para el ejercicio parental. Algunos boicotean lo prescripto por profesionales, maestros o jueces. No se cuenta con sustitutos parentales y los modelos de crianza previo habrían sido también deficitarios. Coinciden que el contexto barrial es un factor de riesgo en la reiterancia de la conducta delictiva en éstos jóvenes, como así también historia de transgresión legal por parte de algunos de los progenitores y señalan la dificultad en la comunicación entre padres e hijos como otro factor de riesgo al igual que la frontera generacional. Las madres tienen escasa diferencia de edad con sus hijos (13, 140 15 años de diferencia). La incondicionalidad en el acompañamiento por parte de las madres cuando los jóvenes están internados lo referencia tres de los cinco entrevistados.

\section{DISCUSIÓN}

De los resultados obtenidos y detallados anteriormente, se puede concluir que el "pensamiento social" de las madres de jóvenes con reiterancia delictiva, muestran un núcleo de representación basado en las emociones positivas que se producen por el hecho de ser madre y la presencia de cualidades personales favorables del sujeto que ejerce ese rol. Podría decirse que hay cierto tipo de reconocimiento de la incumbencia de cualidades a la hora de la 
crianza además de los aspectos emocionales positivos.

Este núcleo representacional, resulta contrastante con la inhabilidad en el ejercicio de las tareas parentales se observan en la crianza de estos adolescentes.

Tanto en la documentación hallada en los legajos institucionales, como en las opiniones de los profesionales expertos dedicados al abordaje integral de la casuística, se delata un proceso de ahijamiento disfuncional. En el mismo, aquellos atributos personales favorables, reconocidos por las progenitoras, no se muestran concretamente en la crianza. Los jóvenes han padecido sistemáticas, graves y continuas vulneraciones de derechos básicos. Las carencias han sido desde la privación de alimentos, de certificación de su identidad, como el sufrimiento de maltrato, o la instigación a cometer delitos. Es decir, que el sentido común sobre lo que puede ser la parentalidad que el grupo resalta desde el afecto positivo y los atributos personales, no se relaciona coherentemente con el producto concreto del ejercicio parental.

El grupo estudiado presenta una clara convergencia con la representación colectiva de la parentalidad y conflicto divergencia en el ejercicio de la tarea concreta hacia dentro del grupo. Considera patrones de pensamiento simbólico coherentes con la expectativa social ampliada, aunque muestra impericia en la práctica relacional.

Por otro lado, las competencias encontradas aluden al cumplimiento de roles institucionales parciales, toda vez que las progenitoras señalan como virtud sobresaliente que sus hijos concurran a la escuela y ellas mantienen contacto de manera constante con la misma.
Descartan otro ámbito de inserción o estimulación de actividades deportivas, religiosas 0 comunitarias. Describen paseos a plazas o parques y casas de otros familiares.

No señalan la constancia del ocio compartido ni tampoco rescatan la co parentalidad ejercida con el progenitor varón.

Se destaca que los vínculos institucionales (Centros de Salud, Escuela) funcionarían como ordenadores de la rutina familiar. En ese sentido, el grupo de pares de jóvenes infractores, encuentra relevancia en la intervención de pautas de socialización, sistemas de información, satisfacción de necesidades, etc., ocupando un lugar preponderante debido probablemente a los requerimientos etáreos y a la carencia parental.

El ejercicio de competencias parciales resultaría insuficiente para cumplimentar funcionalmente la totalidad de los requerimientos parentales, por lo que se infieren áreas disfuncionales.

Los expertos consultados reafirman esta posibilidad toda vez que señalan la incapacidad en la puesta de límites y contención eficaz de conductas socializadoras positivas, encuadradas en la aceptación al orden social.

La relación padre - hijo parece quedarse en los aspectos emocionales positivos sin aceptar el conflicto 0 la confrontación necesaria de la puesta de límites 0 cumplimientos normativos.

Las madres encuestadas también expresan reconocimiento de estos deberes asistenciales y normativos en la primera periferia de la representación social, pero la incapacidad para su puesta en marcha se evidenciaría en los resultados negativos de la crianza. 
Es de destacar compleja tarea de la crianza, involucra no solo cuidados básicos, afectos favorables, mutuos, deberes socializadores (que este grupo reconoce como en el núcleo representacional) sino también la necesidad de su adaptación a la evolución del niño - joven y su ajuste al medio social.

Tanto desde la parentalidad como desde la dinámica construcción de su sentido común, se enfatiza su necesario ajuste a un ecosistema o entorno de desarrollo. De ahí que resulte válido considerar la dificultad en el aprendizaje y ejercicio normativo de la familia y el posible contraste con su comunidad inmediata. Aún más si esa comunidad está focalizada en los pares de los adolescentes vulnerables.

Por ello puede ser válido preguntarse por los modelos de crianza que tuvieron estos progenitores, por el encaje en subculturas 0 subgrupos etáreos en los que no se encuentren alternativas funcionales para replicar, 0 al contrario que enraícen en contextos críticos de mayor vulnerabilidad. Dichos interrogantes intentan contextualizar las respuestas halladas en este estudio, en tanto éstas evidenciarían conflicto entre la pertenencia con la representación social colectiva y la divergencia - variación del ejercicio de la tarea parental en el grupo.

Las madres que participaron en el estudio también muestran capacidad para acompañar y seguir a sus hijos ("hacerles el aguante") cuando éstos ingresan en el sistema de responsabilidad penal juvenil. Podría inferirse cierta adherencia a la pauta normativa, cuando ésta es impuesta desde la autoridad 0 contexto institucional que sobrepasa las fronteras familiares $y$ comunitarias. De esta posibilidad no se da cuenta en este estudio, por lo que también resulta un interrogante para futuras investigaciones.

Teniendo en cuenta la discordancia hallada entre la representación de la parentalidad y su ejercicio concreto, cabe preguntarse si sería válido producir instrumentos de aprendizaje, de guía, de apoyo social específico que favorezcan el desempeño de la tarea de ahijamiento.

Esto, considerando que en el grupo estudiado, las madres de los adolescentes con reiterancia delictiva adhieren a un pensamiento social favorable, tienen afectos positivos, son capaces de "aguantar" 0 acompañar las instancias institucionales de asistencia ante el delito. Probablemente, carezcan de modelos normativos vividos en su propia crianza (interrogante que este estudio no trabaja) por lo que un temprano entrenamiento en programas que favorezcan la maternidad, puede resultar un intento de subvertir la discordancia.

Otra área probable de autogestión positiva, podría ser la implementación de acompañamiento comunitario a través de efectores sociales, agentes sanitarios $y$ referentes barriales que puedan cotidianamente, acompañar la tarea parental.

Estas últimas afirmaciones pueden ser aplicaciones de este trabajo, en las que a partir de este momento se comienza a profundizar e intentar resolver. 


\section{REFERENCIAS}

Abric, J.C. (2001). Prácticas sociales y representaciones. México: Ed.Coyoacán

Barudy, J., Dantagnan, M. (2005). Guía de Valoración de las competencias parentales a través de la observación participante. Material de Investigación. IFIV: Instituto de Formación, Investigación e Intervención sobre la Violencia Intrafamiliar y sus consecuencias. Recuperado de [http://www.buenastareas.com/ensayos/Gu ia-Evaluacion-Competencias-

Parentales/3238182.html].

Barudy, J., Dantagnan, M. (2010). Los desafíos invisibles de ser madre o padre: Fichas de la guía para padres biológicos, acogedores, adoptivos y profesionales de la infancia. Barcelona: Gedisa. Recuperado de [http://www.todoebook.com/LOSDESAFIOS-INVISIBLES-DE-SER-MADREO-PADRE---FICHAS-DE-TRABAJOJORGE-BARUDY-GEDISA-LibroEbookES-SPB0090966.html]

Bayot, A., Hernández, J., De Julián, L. (2005). Análisis factorial exploratorio y propiedades psicométricas de la Escala de Competencia Parental Percibida. Versión para padres/madres (ECPP-P). RELIEVE, Revista Electrónica de investigación y evaluación educativa, 11(2), 113-126.

Becedóniz, C.; Rodríguez, F.; Menéndez, C.; Bringas, C.; Balaña, P., Paíno, S.

(2006). Reincidencia de Menores infractores: investigando factores de la problemática familiar. En Rodríguez, F; Becedóniz, C. (Comp), El Menor Infractor posicionamientos $y$ Realidades (pp.101-118). España: Principado de Asturias.

Casullo, M. M., Fernández Liporace (2008). Percepción sobre estilos e inconsistencia parentales en adolescentes. Estudios de Psicología, 25(1), 3 - 10.

Chan Gamboa, E. C. (2006). Socialización del menor infractor. Perfil psicosocial diferencial en la Zona Metropolitana de Guadalajara, Jalisco. México. (Tesis doctoral) Universidad de Oviedo. Departamento de Psicología. México.

Dekovic, M., Wissink, I.B., Meijer, L. (2004). The role of family and peer relations in adolescent antisocial behavior: comparison of four etnic groups. Journal of adolescence, 27, 497-514.

Demunt, S., Brown S. (2004). Family structure. Family processes ad adolecent delinquency. The significance of parental absence versos parental gender. Journal of research in crime and delinquency. 41 (1), 58-81.

Donini, A. (2007) Sexualidad y Familia. Crisis y desafíos frente al siglo XXI. Buenos Aires: Noveduc.

Dussich, J. (1989). Juvenil delinquency. Definition, carácter and theory. Recuperado de [www.uniovi.es]

Farrington, D. P. (2000). Adolescent violence: Findings and implications from the Cambridge Stude. En G. Boswell (Ed.), Violent Children and Adolescents: Asking the question why. London: Whurr Publishers (pp.19-35).

Farrington, D. P.(2002). Criminology. Criminal Behavior and Mental Health,2 (4):510-516.

Fernández Ríos, L., Gómez Fraquela, J.A, Rodríguez K (1999). La psicología preventiva en la intervención social. Madrid. Síntesis.

García Linares, F., Pelegrina y Lendinez, A. (2002). Los estilos educativos de los padres y la compencia psicosocial de los adolescentes. Anuario de Psicología. 33, 79-95.

González García, C. M., González García, S. I. (2008). Un enfoque para la evaluación del funcionamiento familiar. Revista Mexicana De Orientación Educativa, 6(15), 2-7.

Garrido, V. y. Martínez, M. D. (1998). Educación Social para delincuentes.Valencia: Tirant lo Blanch.

Garrido, V. y López Latorre, M.J. (1995). La prevención de la delincuencia: el enfoque de la competencia social. Valencia: Tirant lo Blanch.

Garrido, V., Stangeland, P. y Redondo, S. (1999). Principios de Criminología. Valencia: Tirant lo Blanc.

González, F. (2010) Representaciones sociales de los alumnos de educación secundaria sobre la escuela y su relación con los resultados académicos. (Tesis de maestría). Universidad Nacional de Cuyo. Facultad de Filosofía y Letras. Mendoza. Argentina.

Graca, M.; Moreira, M.A. y Caballero, C. (2004). Representacoes sobre a Matemática, seu ensino e aprendizagem: um estudo 
exploratório. Revista Investigacoes em Ensino de Ciencias, 9 (1), 37-93.

Gracia, E., Lila, M. y Musitu, G. (2005). Rechazo parental y ajuste psicológico y social en los hijos. Salud mental, 28 (2), 73-81.

Grossi F.; Paínos S.; Fernández J. y Rodríguez F. (2000). Conducta delictiva y ámbito familiar. Revista electrónica iberoamericana de psicología social. $1(1), 8$.

Henggeler, S. () Delinquency in adolescense. NY. Sage Publications.

Jodelet, D. (1986). La representación social: fenómeno, concepto y teoría. En Moscovici, S. (Comp.). Psicología Social II, (pp. 469-494).

Kaufman, S. (1993). The origins of order. Self organizations and selection in evolution. Oxford University Press.

Lopez Coira, M. (1987). Factores familiares y delicuencia en V. Sanchez (Eds) Delincuencia teoría e investigación. Madrid. Abe (pp. 255-274).

Lopez J., Quintana J.C., Cabrera E. y Maiquez M.L. (2009). Competencias parentales en contextos de riesgo psicosocial. Intervención Psicosocial. 18(2), 113-120.

Mazzitelli, C. (2007). El aprendizaje de la Física como reelaboración conceptual a I a luz de algunas teorías psicosociales. Tesis doctoral. Doctorado en Educación Universidad Nacional de Cuyo. MendozaArgentina.

Moscovici, S., y Hewstone, M. (1986). De la ciencia al sentido común. En Moscovici Psicología Social II. Pensamiento y Vida Social. Barcelona. Paidos. 679-710.

Muñoz, L. (2012). Estilos parentales en la familia contemporánea. Conducta delictiva en la adolescencia factores de riesgo y protección. En Valgañón M. (Ed.) Vínculos familiares en transformación. Mendoza. SSyCC ediciones.

Musitu, G., Buelga, S., Lila, M. y Cava. M. J. (2001). Familia y adolescencia. Madrid: Síntesis.

Musitu, G. y Cava, M. J. (2001). La familia y la educación. Barcelona: Octaedro.

Musitu, G. y García, F. (2001). Escala de socialización parental en la adolescencia. Madrid: TEA.

Musitu, G. (2002). Las conductas violentas de los adolescentes en la escuela: el rol de la familia. Aula abierta, 79: 109-138.
Musitu, G. y García, F. (2004). Las consecuencias de la socialización en la cultura española.

Psicothema, 16, 288-293.

Musitu, G., Estévez, E., Jiménez, T. y Herrero, J. (2007). Familia y conducta delictiva y violenta en la adolescencia. En S. Yubero, Larrañaga, E. y Blanco, A. (Coords.), Convivir con la violencia (pp. 135-150). Cuenca: Ediciones de la Universidad de Castilla-La Mancha.

Paíno, S.G., Rodríguez, F.J. y Garrido, V. (1996). Socialization, drug abuse and criminal career. VI European Conference on Psychology and Law,agosto, Siena (Italia).

Paíno, S.G. y Rodríguez, F.J. (1998). Socialización y delincuencia. Un estudio en la prisión de Villabona (Asturias). En V. Garrido y $M^{a}$ D.Francés. Educación Social para Delincuentes. Valencia: Tirant lo Blanch (pp. 97-136).

Paterna, C., Martínez, M. C. y Vera, J. J. (2003). Psicología social. Madrid: Pirámide.

Peñalva, C. (2001). Evaluación, $N$ del funcionamiento familiar por medio de la "Entrevista estructural" (Español). Salud Mental, pp. 32-42. Recuperado de [http://new.medigraphic.com/cgibin/resume n.cgi?IDREVISTA=81\&IDARTICULO $=22365 \&$ IDPUBLICACION=2273]

Petracci, M. \& Kornblit, A. (2007). Representaciones sociales: Una teoría metodológicamente pluralista en Kornblit A (comp). Metodologias cualitativas en Ciencias Sociales. Bs. As. Biblos.

Rodrigo López, M. ${ }^{a}$ J.; Martín Quintana, J. C.; Cabrera Casimiro, E. ; Máiquez Chaves, M. ${ }^{a}$ L. (2009). Las Competencias Parentales en Contextos de Riesgo Psicosocial. Intervención Psicosocial, 18,2, 113-120.

Rodríguez, A; Torrente, G. (2003). Interacción familiar y conducta antisocial. Boletín de Psicología, 78, 7-19.

Rutter, M., Giller, H. y Hagell,A. (1998). Antisocial behavior by young people. Ney York: Cambridge University Press.

Ryan, E.P. y Redding, R.E. (2004). A review of mood disorders among juvenile offenders. Psychiatric Services, 55 (12), 1397-1407.

Valgañón, M. (Ed.) (2012). Vínculos familiares en transformación. Estilos, modelos y competencias parentales. Mendoza. SSyCC ediciones. 
Vázquez González, C.(2003). Teorías criminológicas sobre delincuencia juvenil. En Vázquez Gónzalez, C.(Ed). Delincuencia juvenil. Consideraciones penales y criminológica (pp. 63-119). Madrid: Colex.

Vega (1987 ). La escuela. En F. de la Garza La cultura del menor infractor. Mexico. Trillas. 